CNU-AST-96

\title{
Numerical Simulations of Standing Shocks in Accretion Flows around Black Holes: A Comparative Study"
}

\author{
Diego Molteni \\ Istituto di Fisica, Via Archirafi 36, 90123 Palermo Italy; \\ e-mail: molteni@gifco.fisica.unipa.it \\ Dongsu Ryu \\ Dept. of Astronomy \& Space Sci., Chungnam National Univ., Daejeon 305-764, Korea; \\ e-mail: ryu@sirius.chungnam.ac.kr \\ and \\ Sandip K. Chakrabarti \\ Tata Institute of Fundamental Research, Bombay 400005, India; \\ e-mail: chakraba@tifrc2.tifr.res.in
}

\begin{abstract}
We compare the results of numerical simulations of thin and quasi-spherical (thick) accretion flows with existing analytical solutions. We use a Lagrangian code based on the Smooth Particle Hydrodynamics (SPH) scheme and an Eulerian finite difference code based on the Total Variation Diminishing (TVD) scheme. In one-dimensional thin flows, the results of the simulations, with or without shocks, agree very well with each other and with analytical solutions. In two-dimensional thick flows, the general features, namely the locations and strengths of centrifugal and turbulent pressure supported shocks, centrifugal barriers, and the funnel walls which are expected from analytical models, agree very well, though the details vary. Generally speaking, the locations of the shocks may be better obtained by SPH since the angular momentum is strictly preserved in SPH, but the shocks themselves are better resolved by TVD. The agreement of these code test results with analytical solutions provides us with confidence to apply these codes to more complex problems which we will discuss elsewhere.
\end{abstract}

Subject headings: Accretion, Accretion Disks - Black Hole Physics - Hydrodynamics Methods: Numerical - Shock Waves

\footnotetext{
${ }^{1}$ The Astrophysical Journal in press
} 


\section{INTRODUCTION}

A great deal of interest is present in the astrophysical community in understanding the accretion flows around black holes, both analytically as well as numerically. The scope of analytical works is usually very limited, since these are devoted to obtain only steady state solutions or to study stability properties of these solutions based on local or in rare cases, global stability analysis. However, astrophysical systems are hardly in a steady state. Fluctuations and flickering of radiation emitted from these systems constantly remind us of time-variations of the dynamical and thermo-dynamical quantities. The variations can take place on times scales of few micro-seconds

to few years. In order to understand such systems it is necessary to perform numerical simulations of these systems. On the other hand, numerical techniques of writing a code are not unique, and not all techniques are equally accurate. To be able to rely on a particular numerical scheme, it is required that numerical results be tested against analytical solutions, stationary or non-stationary, of presumably simpler systems which are as similar as possible to the actual systems that will be eventually studied by the code. Testing of a code is thus an essential component of a numerical study of the long time behavior of a system. In the present paper, we shall compare two codes, one is a Lagrangian code based on the Smoothed Particle Hydrodynamics (SPH; Monaghan, 1985, 1992) and the other is an Eulerian code based on the Total Variation Diminishing scheme (TVD; Harten 1983; Ryu et al. 1993, 1995) appropriately modified to study cylindrically symmetric problems. The tests are carried out against some well known non-linear solutions of axisymmetric transonic accretion disks around a black hole. We show that both of our codes agree very well with the analytical results. Armed with the confidence that our codes are reasonably good for study of time dependent flows, we present more complex behavior of time dependent accretion flows in our next papers (Ryu, Chakrabarti, \& Molteni 1996; Molteni, Chakrabarti, \& Ryu 1996).

Accretion flows are important ingredients in many astrophysical systems which involve mass transfer from one object to another (such as in a binary system) or set of objects to another (such as in a galactic center). There is ample evidence in the literature that accretion disks exist in systems ranging from CVs, LMXBs on a small scale, to AGNs and Quasars on a large scale. Standard disk models of Shakura \& Sunyaev (1973) and Novikov \& Thorne (1973) assume Keplerian distribution of accreting matter. Here, the inner edge of the disk is chosen to coincide with the marginally stable orbit located at three Schwarzschild radii $\left(r_{i}=3 r_{g}\right.$, where $r_{g}=2 G M_{B H} / c^{2}$ is the Schwarzschild radius of a black hole of mass $M_{B H}$ ). These disk models are clearly incomplete, since the inner boundary condition on the horizon was not taken care of. Because flow at the inner boundary (namely, the horizon) is bound to be supersonic, in order to satisfy this condition, it would start to deviate from a Keplerian disk and enter into the black hole horizon through the sonic surface or sonic horizon even if it originally starts from a Keplerian disk. Paczyński \& Bisnovatyi-Kogan (1981) generalized the equations governing these flows and solved these equations only close to the inner edge of the disk using numerical methods. It was demonstrated that indeed the flow enters the black hole supersonically.

This is not the full story. Liang \& Thompson (1980) pointed out that unlike a Bondi flow, 
where only one saddle type sonic point is allowed (e.g., Thompson \& Stewart, 1985), rotating accretion and winds can have two saddle type sonic points. After the flow passes through the outer sonic point, the centrifugal force can virtually stop the rotating supersonic flow after forming a shock close to a black hole and restart its journey once more to pass through the inner sonic point and to satisfy the inner boundary condition on the horizon (Chakrabarti 1989, hereafter C89). Extensive analytical results are now available to understand the nature of these transonic solutions which contain standing shocks (C89) and recently, it has been shown that these generalized accretion flows could be responsible for the hard and soft state transitions in the black hole candidates (Chakrabarti \& Titarchuk, 1995), or even responsible for quasi-periodic oscillation (QPO) of the hard X-rays from the black hole candidates (Molteni, Sponholz \& Chakrabarti 1996, hereafter MSC96; Ryu et al. 1995). In latter work, one invoked resonance oscillation of the shocks to explain QPOs. Recently, we found that the oscillation is more generic in that it is related to the transient shock formation in a wider parameter space (Ryu, Chakrabarti, Molteni, 1996) thus suggesting persistent QPOs even when accretion rate varies by a large factor.

Numerical simulations of the shocks in accretion flows, however, preceded these analytical studies. The first numerical attempt to study the behavior of rotating accreting matter around black holes was made by Wilson (1978). An Eulerian, fully general relativistic, first-order backward space difference technique was used. The spatial resolution was low and the solution was evolved only upto $\sim 100 G M / c^{3}$. It was shown that large angular momentum accretion is accompanied by shocks which traveled outwards. This code was later improved upon, with the number of grid points as well as the evolution time orders of magnitude higher. A series of very important simulations were made with this code to show that thick accretion disks can indeed form in inviscid flows (Hawley, Smarr \& Wilson 1984, 1985). These simulations also confirm the results of Wilson (1978) that non-steady shocks are formed which travel outward. From the post-shock flow, a very strong wind is generated which is hollow and hugs the funnel wall. Due to the inviscid nature of the simulation, the centrifugal force kept the flow away from the axis of symmetry. A better understanding of the shock formation in the disk emerged only after the analytical works and numerical works (C89, Chakrabarti \& Molteni 1993, hereafter CM93; Molteni, Lanzafame \& Chakrabarti 1994, hereafter MLC94) were able to show that standing shocks can not only form in accretion and winds, they are stable as well. They also form very close to the location where analytical works predict them. Subsequently, these numerical simulations were continued in the case of flows around a Kerr black hole (Sponholz \& Molteni, 1994, hereafter SM94) and in viscous transonic disks (Chakrabarti \& Molteni, 1995; hereafter CM95).

Most of these simulations (CM93, CM95, MLC94, SM94) were carried out using the Smoothed Particle Hydrodynamics (SPH) technique (Monaghan 1985, 1992). It is found to be a very powerful tool for astrophysical applications, particularly when proper care is taken to ensure the energy and angular momentum conservation. This was done by implementing the code in cylindrical coordinates where each particle is assumed to be an axisymmetric torus (Molteni \& Sponholz, 1993). The results obtained by these simulations generally agree with the analytical works, in 
terms of the shock location and strengths, etc. However, the SPH methods often are charged of being unduly dissipative and it is essential to quantify the behavior of SPH by comparing with analytical work, as well as other finite different methods. Recently, Ryu et al. (1995) have considered a problem related to accretion of sub-Keplerian inflow very similar to MLC94 using a completely different code. This code is an Eulerian finite difference code based on the Total Variation Diminishing (TVD) scheme developed by Harten (1983). The code is found to conserve energy and angular momentum sufficiently accurately, but it has not been tested yet against analytical works in the non-linear solutions of accretion processes of quasi-spherical flows. In the present paper, we shall compare the results from the TVD code as well as the SPH code with the analytical solution of accretion flows in the black hole geometry.

A few other comparisons between SPH and finite difference codes have already been made in the literature. Steinmetz \& Müller (1993) compared one-dimensional plane-parallel hydrodynamic shock solutions and spherical cloud collapse and suggested some improvements in the SPH technique to minimize dissipation. Davies et al. (1993) compared collisions of a main sequence star with a white dwarf and showed a satisfactory performance of SPH as well as the finite difference code. Laguna, Miller \& Zurek (1993) compared a general relativistic SPH code for one-dimensional plane-parallel shock solutions and spherical inflows (with and without pressure) with an analytical model and found a very good agreement. Kang et al. (1994) compared six different codes among each other including the TVD code and two versions of SPH applied to cosmological hydrodynamics, but did not directly compare numerical results to any analytical solution. However, comparisons of codes and analytical work in relation to rotating inflows have not been performed yet. We set out to do this here.

The plan of the present paper is the following. We very briefly present the general procedure of obtaining analytical solutions (with or without shocks) in the next Section. In $\S 3$, we present model equations used in the time dependent simulations and describe the numerical schemes briefly. In $\S 4$, we present one-dimensional solutions of both codes and discuss merits and demerits. In $\S 5$, we present comparisons of simulations in two-dimensions. Finally, in $\S 6$, we make concluding remarks.

\section{MODEL EQUATIONS FOR ANALYTICAL STUDY}

We assume a thin, rotating, adiabatic accretion or wind near a black hole. We take the Newtonian model for the non-rotating central compact object as given in terms of the Paczyński $\&$ Wiita (1980) potential. We also assume a polytropic equation of state for the accreting (or, outflowing) matter, $P=K \rho^{\gamma}$, where, $P$ and $\rho$ are the isotropic pressure and the matter density respectively, $\gamma$ is the adiabatic index (assumed in this paper to be constant throughout the flow, and is related to the polytropic index $n$ by $\gamma=1+1 / n$ ) and $K$ is related to the specific entropy of the flow $s$. We assume that entropy, and thus $K$ can vary along a flow line only at a shock. We ignore dissipation, so the specific angular momentum $\lambda \equiv x v_{\theta}$ is also constant 
everywhere. A complete solution of the stationary model requires the equations of energy, momentum and mass conservation supplemented by the transonic conditions at the critical points and the Rankine-Hugoniot conditions at the shock. The general procedure followed is the same as is presented in Chakrabarti (1989). In this Section, we present equations which are the same as in Chakrabarti \& Molteni (1993) where vertical thickness is chosen to be constant. The last assumption allows one to test the TVD and SPH codes in one-dimension.

We use the mass of the black hole $M$, the velocity of light $c$, and the Schwarzschild radius $x_{g}=2 G M / c^{2}$ as the units of mass, velocity and distance respectively. The dimensionless energy conservation law can be written as

$$
\mathcal{E}=\frac{v_{x}^{2}}{2}+\frac{a^{2}}{\gamma-1}+\frac{\lambda^{2}}{2 x^{2}}+g(x)
$$

Here $g(x)$ is the radial force potential, which in the pseudo-Newtonian model takes the form: $g(x)=-1 / 2(x-1)^{-1}$. Here, $v_{x}$ and $a$ are the non-dimensional radial and the sound velocities, $x$ is non-dimensional radial distance. Apart from an unimportant geometric factor, the mass conservation equation is given by

$$
\dot{M}=v_{x} \rho x h_{0},
$$

where $h_{0}$ is the constant half-thickness of the flow. The shock conditions which we employ here are the following (subscripts '-' and '+' refer to quantities before and after the shock): The energy conservation equation

$$
\mathcal{E}_{+}=\mathcal{E}_{-}
$$

the pressure balance condition

$$
P_{+}+\rho_{+} v_{x+}^{2}=P_{-}+\rho_{-} v_{x-}^{2}
$$

and the mass conservation equation

$$
\dot{M}_{+}=\dot{M}_{-} .
$$

In order to have a shock, the flow must be supersonic, i.e., the stationary flow must pass through a sonic point. The sonic point conditions are derived following the usual general procedure (C89) and one obtains the sonic point conditions as

$$
v_{c}\left(x_{c}\right)=a_{c}\left(x_{c}\right)
$$

and

$$
a_{c}^{2}\left(x_{c}\right)=\frac{\lambda_{K}^{2}-\lambda^{2}}{x_{c}^{2}}
$$

The subscript $c$ denotes quantities at the critical points. Here, $\lambda_{K}(x)=(x / 2)^{1 / 2} /(1-1 / x)$ is the Keplerian angular momentum. Eqs. (1), (2), (3a-c), (4a-b) are solved simultaneously to obtain the full set of solutions which may include shocks.

Whereas above equations are valid for a steady state flow with constant height, they are easily generalized for a flow in vertical equilibrium. In this case, the flow height $h_{0}$ of Eq. (2) is 
replaced by the flow thickness $H(x) \sim a x^{1 / 2}(x-1)$ appropriate for a disk in vertical equilibrium and the shock condition is imposed over the integrated pressure and density. Details are in C89 and we do not discuss them here.

It is not necessary that a flow contains shocks at all. Usually after the flow deviates from a Keplerian disk, it may pass through the inner sonic point straight away into the black hole, or, through the outer sonic point or both (when shocks join these two types of flows). The actual fate of the flow depends on two parameters, the specific angular momentum and the specific energy (C89). In fact, even the outer sonic point may not exist for flows with a large polytropic index (e.g. $\gamma=5 / 3$ ) unless the flow is very thin (MSC96). Most general and complete global solution topologies of viscous transonic flows (Chakrabarti 1996, hereafter C96, and references therein) show more complex behavior since the presence or absence of transonic solution depends on efficiencies of cooling and heating processes and well as the viscosity.

\section{EQUATIONS AND SCHEMES USED IN THE NUMERICAL STUDY}

The TVD code used in this paper is exactly same as that used and described in details in Ryu et al. (1995), except for the form of the gravitational force which is modified here according to pseudo-Newtonian potential (Paczyński \& Wiita, 1980). The scheme was originally developed by Harten (1983). It is an explicit, second order accurate scheme which is designed to solve a hyperbolic system of the conservation equations, like the system of the hydrodynamic conservation equations. It is a nonlinear scheme obtained by first modifying the flux function and then applying a non-oscillatory first order accurate scheme to get a resulting second order accuracy. Thus the key merit of this scheme is to achieve the high resolution of a second order accuracy, while preserving the robustness of a non-oscillatory first order scheme.

The equations solved numerically with the TVD code are written in vector form using non-dimensional units as:

$$
\frac{\partial q}{\partial t}+\frac{1}{x} \frac{\partial\left(x F_{1}\right)}{\partial x}+\frac{\partial F_{2}}{\partial x}+\frac{\partial G}{\partial z}=S,
$$

where the state vector is

$$
q=\left(\begin{array}{c}
\rho \\
\rho v_{x} \\
\rho v_{\theta} \\
\rho v_{z} \\
E
\end{array}\right)
$$


the flux functions are

$$
F_{1}=\left(\begin{array}{c}
\rho v_{x} \\
\rho v_{x}^{2} \\
\rho v_{\theta} v_{x} \\
\rho v_{z} v_{x} \\
(E+p) v_{x}
\end{array}\right) \quad F_{2}=\left(\begin{array}{c}
0 \\
p \\
0 \\
0 \\
0
\end{array}\right) \quad G=\left(\begin{array}{c}
\rho v_{z} \\
\rho v_{x} v_{z} \\
\rho v_{\theta} v_{z} \\
\rho v_{z}^{2}+p \\
(E+p) v_{z}
\end{array}\right)
$$

and the source function is

$$
S=\left(\begin{array}{c}
0 \\
\frac{\rho v_{\theta}^{2}}{x}-\frac{\rho x}{2\left(\sqrt{x^{2}+z^{2}}-1\right)^{2} \sqrt{x^{2}+z^{2}}} \\
-\frac{\rho v_{x} v_{\theta}}{x} \\
-\frac{\rho z}{2\left(\sqrt{x^{2}+z^{2}}-1\right)^{2} \sqrt{x^{2}+z^{2}}} \\
-\frac{\rho\left(x v_{x}+z v_{z}\right)}{2\left(\sqrt{x^{2}+z^{2}}-1\right)^{2} \sqrt{x^{2}+z^{2}}}
\end{array}\right) .
$$

Here, energy density $E$ (without potential energy) is defined as, $E=p /(\gamma-1)+\rho\left(v_{x}^{2}+v_{\theta}^{2}+v_{z}^{2}\right) / 2$.

Note that, in the case of axisymmetric flow without viscosity, the azimuthal momentum equation is simply the conservation of angular momentum

$$
\frac{d \lambda}{d t}=0
$$

So for the problem considered in this paper, the equation for the azimuthal momentum can be decoupled from the rest. But the TVD code used in this paper was designed to solve the whole set of the equations including that for the azimuthal momentum, so as to be able to handle the general cases (such as viscous flows) where the angular momentum could be transported from one region to another. As a result, the TVD calculations do not conserve the angular momentum exactly but have an error typically less than a couple of percent in the angular momentum conservation.

The equations governing the SPH simulations are already provided in CM93, and MLC95, and we do not repeat them here.

In what follows, we present the results of the calculations using the SPH and TVD codes comparing them with the analytical work in one-dimensional cases and with each other in two-dimensional cases. 


\section{COMPARISON OF NUMERICAL SIMULATION RESULTS IN ONE-DIMENSION}

Physically, a shock can form when the rotating flow is close to the centrifugal barrier. However, because the flow can have a significant pressure, it is not essential that the angular momentum should be so high as to hit an actual barrier. That is, the flow angular momentum could be much lower than the marginally stable value ( $\sim 1.83$ in our units). In the current simulation, we choose $\lambda=1.8$, slightly below the marginally stable value. In the following comparison, we present the TVD calculation with 512 grid points between 0 to $x=50$ (Fig. 1 and Fig. $2 \mathrm{~b}$ ) or 0 to $x=90$ (Fig. $2 \mathrm{~b}$ ), and the SPH calculation with $\sim 560$ particles (of size $h=0.3$ ) in Fig. 1 and Fig. 2a, and $\sim 1080$ particles (of size $h=0.25$ ) in Fig. 2b. Matter is injected at the outer boundary located at $x=50$ or 90 subsonically with the velocity and sound speed obtained from the analytical solution. At the inner boundary, an absorption boundary condition is used to mimic the black hole horizon. In the TVD simulations the inner edge is kept at $x=1.5$, and in the SPH simulations it is kept at $x=1.25$. (Horizon is at $x=1$ ). We choose the adiabatic index $\gamma=4 / 3$, which is appropriate for very relativistic flows or very optically thick radiation pressure dominated flows. This index is also valid for optically thin flows (perhaps in the presence of magnetic fields) with some cooling effects (such as the Comptonization of external soft photons). The goal was to see to what extent shocks are captured by the flow. In the TVD simulations we have tested the one dimensional code with 128, 256, 512 and 1024 uniformly spaced grid points whereas in the SPH simulations we have tested the code with various particle sizes $h$. We find the result of varying resolutions to agree with each other very well, though the thickness of the shock could vary with the grid resolution. Thus, we are convinced that the code is definitely converged at the grid resolution presented.

Analytical solution of a given problem requires two conserved quantities, such as the specific energy $\mathcal{E}$ and angular momentum $\lambda$. The entire solution, including the locations of sonic points and the shock, is determined by these two parameters (C89). If the flow is viscous, the viscosity parameter is also required (CM95, C96 and references therein) and in return, the location where the flow joins with a Keplerian disk comes out as an eigenvalue. In the first test case, we choose the energy parameter $\mathcal{E}=0.0363$ with angular momentum $\lambda=1.8$ and the outer boundary at $x=50$. In Fig. 1, we superimpose the numerical simulation results on the analytical result. For clarity near the inner egde we only show the region $x \leq 35$ (for $x>35$ agreement is perfect). In this case, the flow starts out subsonically, presumably from a Keplerian disk, then enters through the outer sonic point, passes through the shock and then subsequently passes through the inner sonic point before entering the black hole. The upper panel shows the variation of density and the lower panel shows the variation of the Mach number with the radial distance $(x)$. The solid curve is the analytical solution, whereas the long dashed curve and the short dashed curves are the results of the TVD calculation and the SPH calculation respectively. The agreement is clearly very good. The shock is thicker in the SPH calculation than in the TVD one. Also the density peak around $x \sim 3.5$ is slightly underestimated in the SPH calculation. 
In the analytical solution, the locations of the outer sonic point $x_{o}$, the inner sonic point $x_{i}$ and the stable shock $x_{s 3}$ (using the notations of C89, CM93), for the flow parameters mentioned above, are at 27.9, 2.563 and 7.8896 respectively. In the TVD simulation, these numbers were 27.97, 2.57 and 7.98 respectively, while in the SPH simulation we find these numbers to be 26.78, 2.571 and 8.46 respectively. The exact shock location is probably in error since the shock is somewhat diffuse, more so in the SPH simulation.

It is known that for every angular momentum (within a range) at the outer boundary, there is a range of specific energy of the flow, for which the shock conditions are satisfied in accretion flows (see, Fig. 4 of C89). If the energy is smaller than the lower limit of the range, the flow only passes through the outer sonic point and no shocks are present. Similarly, if the energy is higher than the upper limit of the range, the flow would only pass through the inner sonic point and the question of shock formation does not appear. This is true even if the flow of outside the shock regime has two saddle type sonic points. We present now two such examples where the flow can pass only through one sonic point at a time. In Fig. 2a, we present a comparison of solutions which has only the inner sonic point at $x_{i}=2.46$. The energy parameter in this case is $\mathcal{E}=0.07$ with $\lambda=1.8$. The outer boundary for the simulation is chosen at $x=50$. In Fig. 2b, we present the comparison of solutions with the outer sonic point located at $x_{o}=77.615$. The energy parameter in this case is $\mathcal{E}=0.015$, with $\lambda=1.8$. The outer boundary for the simulation is chosen at $x=90$. Again the plots show only the region with $x \leq 35$ for clarity of comparison close to the black hole. The solid, long-dashed and short dashed curves are the analytical, TVD and SPH solutions respectively. The TVD results agrees very well with the analytic solutions. The agreement of the SPH results is clearly acceptable, although not quite as good as that of the TVD ones.

\section{COMPARISON OF SHOCK STUDIES IN TWO-DIMENSIONAL FLOWS}

A realistic accretion disk is expected to be three-dimensional, of course. Assuming axisymmetry, the problem is reduced to two-dimensions. It is difficult to solve analytically the problem in full generality even in two-dimensions. The nearest case that is solved analytically is the inflow which is in instantaneously in vertical equilibrium (C89, C96). A comparison of such an analytical solution with the two-dimensional SPH solution (MLC94) suggests that the flow displays more complex behavior, because of the presence of turbulence in the backflow which is produced as the flow hits the centrifugal barrier. The turbulent pressure seems to be comparable to other pressure effects, such as thermal and ram pressure, and it changes the location of the shock significantly. It also increases the chance of shock formation. The parameter space within which steady shocks may develop appeared to be much larger in presence of turbulence (MLC94).

For the simulations of the two-dimensional quasi-spherical flow, we choose to place the outer boundary at $x=50$. The height of the disk is 56 at the outer boundary (assuming vertical equilibrium). The specific angular momentum of the flow is chosen to be $\lambda=1.65$. The radial (in the spherical sense) velocity components is chosen to be constant at all height 
$v_{r}=\left(v_{x}^{2}+v_{z}^{2}\right)^{1 / 2}=0.068212$. We also choose the adiabatic index $\gamma=4 / 3$ as before. On the equatorial plane, the energy parameter chosen is $\mathcal{E}=0.004$. This implies a sound speed of $a=0.061463$ on the equatorial plane. We employ an isothermal outer boundary condition (namely, the same sound speed at all height). The matter is injected at the outer boundary in both the codes and is absorbed at the inner edge in the same way as in the one-dimensional case.

With the specific energy and angular momentum chosen as above $(\mathcal{E}=0.004, \lambda=1.65)$, according to the model flow in vertical equilibrium (C89) there is no analytical shock solution in the equatorial plane in the absence of turbulence pressure. However, the specific energy increases with vertical height (with the decrease in potential energy) and the flow comes within the regime of shock formation even without the turbulence (Fig. 8c of C89). Thus a shock is naturally expected at a higher elevation. Since the gravitational force decreases with vertical height, it requires less centrifugal force to form a shock. Hence the shock is expected to be at a larger distance $x$ at higher elevations. In other words, it is expected that the shock would be bent outward as one deviates away from the equatorial plane.

There are two important features of a rotating, thick accretion flow: one is the funnel wall and the other is the centrifugal barrier. Roughly speaking, the funnel wall is described by the surface $\left(x_{f}, z_{f}\right)$ which is the surface of vanishing effective potential

$$
\Phi_{e f f}=-\frac{1}{2\left(r_{f}-1\right)}+\frac{\lambda^{2}}{2 x_{f}^{2}}=0,
$$

where, $r=\left(x^{2}+z^{2}\right)^{1 / 2}$ is the spherical distance. On the other hand, the centrifugal barrier $\left(x_{c f}, z_{c f}\right)$ is governed by the competition between the centrifugal force and gravitational force:

$$
-\frac{1}{2\left(r_{c f}-1\right)^{2}}+\frac{\lambda^{2}}{x_{c f}^{3}}=0 .
$$

Matter stays bound up to the effective potential barrier, or the so-called funnel wall. However, the centrifugal force dominates over gravity between the funnel wall and the centrifugal barrier. The accretion flow first hits the effective potential barrier and piles up behind it. A part of the flow moves backward, and its interaction with the infalling matter causes turbulence. Combined effects of thermal pressure $P$ (whose presence is mainly due to centrifugal force anyway) and turbulent pressure $P_{t}$ 'brakes' the incoming flow which then undergoes a shock transition. If the piled up 'atmosphere' of the black hole thickens with time, either due to a very strong centrifugal barrier or because of faster viscous transport of angular momentum in this region, the shock propagates outwards and is not steady (CM95). But in presence of smaller viscosity the shock can be steady and forms another surface where the pressure balance condition (Eq. 3b) is satisfied in the thick flow:

$$
P_{+}(x, z)+P_{t+}+\rho_{+}(x, z) v_{+}(x, z)^{2}=P_{-}(x, z)+P_{t-}+\rho_{-}(x, z) v_{-}(x, z)^{2} .
$$

As mentioned earlier, the shock is expected to bend outwards, roughly following the contour of the centrifugal barrier (Eq. 8). 
Below, we show that our simulations using two codes capture all the three surfaces described by Eqs. 7-9 very unambiguously.

The TVD calculation for the two-dimensional flow has been done with $128 \times 256$ cells in a box of $50 \times 100$. The SPH calculation has been done with $\sim 12800$ particles in the same box. The results show that, after the initial set-up period with $t \lesssim 5 \times 10^{3}$, the matters settles on a steady-state solution.

Figs. 3(a-b) plot the density contours of the TVD (Fig. 3a) and SPH (Fig. 3b) calculations in a box of $50 \times 50$ at $t=2 \times 10^{4}$. Both the solutions reached steady states (in large scale behavior) much before this time. At the outer boundary, density is chosen to be unity in both the codes. Within the box of $50 \times 50$, the number of TVD grids are $128 \times 128=16384$, out of which a couple of thousands are basically empty (within the funnel wall). In SPH calculation, on the other hand, within $50 \times 50$ box there were 11229 particles, all participating in the dynamics of the flow. Thus, the resolutions in the two calculations are probably comparable, though SPH resolution is higher closer to the black hole due to accumulation of more particles there. In both the figures, the contour level of the minimum density is 0.0261 . Successive contours have a density ratio of 1.2. Only some of the contours are marked for clarity. The plots of both the simulations have the same contour levels.

There are clearly general agreements between the two calculations. Both the simulations show clear presence of the funnel wall. (Ruggedness of the contours in Fig. 3b is an artifact of interpotation of SPH results on equal spaced grids required for contour plotting.) The most visible structure is the quasi parabolic stationary accretion shock which runs vertically and bends outwards as discussed above. In the SPH simulation, the angular momentum of each particle is preserved by construction (as each particle is described by an axisymmetric torus), but in the TVD code it can be redistributed due to numerical diffusion. A closer examination of the results has shown that the TVD code preserves angular momentum to within a couple of percent. In fact, it seems to have a little less average angular momentum near the shock. This may make the centrifugal force somewhat weaker and the paraboloidal shock may be located inwards because of this. Such a dependence of the shock location with angular momentum also conforms with analytical understanding (e.g., Fig. 8c of C89). Note that due to general reduction of angular momentum, matter tended to pile up more closer to the black hole in TVD simulation. This is evidenced by a comparison of the locations of a given density contour on both the figures.

Both simulations show the presence of another oblate spheroidal shock near the equatorial plane. Both touch the equatorial plane $(z=0)$ at roughly the same location, at $x \sim 24$ in the TVD simulation and at $x \sim 26$ at the SPH simulation. This shock near the equatorial plane is a result of the combination of backflow and pressure effects. Whereas in TVD code the two shocks (the parabolic and the spheroidal shocks) cross each other possibly because the paraboloidal shock may have moved inwards, in the SPH results they just touch on the equatorial plane. Away from the equatorial plane, this shock is formed by the accelerated post paraboloidal shock flow coming 
from the higher elevation. In general, the shocks are better resolved in TVD than in SPH.

Figs. 4 plot the velocity fields of the TVD calculation (Fig. 4a) and the SPH calculation (Fig. 4b). In the TVD plot, the cells with the density higher than 0.0261 of the inflow density at the outer boundary have been shown with arrows placed on alternate grid points for clarity, while in the SPH plot all densities are used. But arrows have been put on every fifth particle (of the final sequence) for clarity. We also superimpose two surfaces, the funnel wall described by Eq. 7 (inner curve) and the centrifugal barrier by Eq. 8 (outer curve) on these velocity fields. In the TVD calculations some inflow material close to the funnel wall is mixed with the background material of zero angular momentum, resulting in material with smaller angular momentum than the inflow material. This is a disadvantage of grid based finite difference codes. On the other hand, in the $\mathrm{SPH}$ code the inflow material is represented by particles and there is no need for any background material. As a result, in the SPH plot all material is outside the funnel wall, while in the TVD plot some matter penetrates inside the funnel wall. The presence of the prominent centrifugal barrier in the SPH plot is probably due to the strict conservation of angular momentum in SPH. However, since the shock is better resolved in TVD, the velocity vectors reflect better the shock transition of the parabolic accretion shock (from supersonic long vectors to subsonic short vectors). The back flow toward the positive $x$-direction inside the vortex is clearly visible in the TVD calculation, but does not exist in the SPH calculation. The clumsiness of the arrows in SPH closer to the hole is because too many particles are present there. But the flow is perfectly smooth and well behaved.

The test calculation with a lower resolution using $64 \times 128$ cells using a TVD code has shown the same structures as those in Figs. 3a and $4 \mathrm{a}$ with the positions of shocks and vortex agreeing reasonably well. SPH simulation has also been carried out with larger particle size $h \sim 1$ and produce thicker shock wave, but at the same location. Although this is partly because our calculation has produced relatively simple structures, we think that the two-dimensional calculation presented in this paper show converged results.

\section{CONCLUDING REMARKS}

In the present paper, we have compared for the first time analytical results of inviscid transonic flows with the results of two hydrodynamic codes based on completely different mathematical techniques. We have found that, roughly both the codes agree with each other and with analytical solutions quite well, though the details vary. In one-dimensional calculations, both the codes agree very well with the analytical solution of density and Mach number distributions. They reproduce the locations of the sonic points and the shocks with high accuracy. For the first time, we have also been able to actually follow subsonic inflow at the outer boundary while it crosses the outer sonic point in its inward journey with (Fig. 1) or without shocks (Fig. 2). In two-dimensional calculations, both the codes have shown the formation of the funnel wall and the centrifugal and turbulent pressure supported shocks. In TVD calculation angular momentum is conserved within a couple of percent, which might have caused the accretion shock to form slightly 
inside. The SPH technique conserves angular momentum strictly. On the other hand, in the TVD calculation the shocks are well resolved while in the SPH calculation the shocks are wider. In two-dimensions, a complete analytical theory does not exist. However, extrapolated understanding of one-and-a-half dimensional flow behavior (C89), such as the curved vertical shock, funnel wall, and the centrifugal barrier, agrees well with what we see in our simulations. Based upon this experience, we believe that just as the comparison of plane-parallel shock results constitutes a definitive test for accuracy of a code written in the Cartesian coordinate, any code written in spherical or cylindrical co-ordinate should be tested against our shock solutions to measure the accuracy of such codes.

A realistic two-dimensional flow at the outer boundary located at a large distance may either be sub-Keplerian (as probably in active galaxies) or may be Keplerian (in binary systems and active galaxies). But in both the cases the inflow at the outer boundary is expected to be subsonic. We have already presented examples of stable one-dimensional solutions, with or without shocks in our simulations, which are subsonic to begin with. Fully two-dimensional simulations with the subsonic outer boundary condition (which necessarily means that the boundary be located much farther out beyond the outer sonic point) require more efforts and the computational time. Similarly, our present simulations were done for parameter ranges producing steady solutions. In reality, some solutions may be inherently time dependent showing oscillations of various time scale (as in MSC96, Ryu et al. 1995). Such simulations are presently in progress and will be described in a subsequent paper.

The work by DR was supported in part by the Basic Science Research Institute Program, Korean Ministry of Education 1995, Project No. BSRI-95-5408. The authors thank the referee for suggesting improvements in overall presentation of the paper. 


\section{REFERENCES}

Chakrabarti, S. K., 1989, ApJ, 347, 365 (C89).

Chakrabarti, S. K., 1996, ApJ, (June 20th issue, in press).

Chakrabarti, S. K., \& Molteni, D., 1993, ApJ, 417, 671 (CM93).

Chakrabarti, S. K., \& Molteni, D., 1995, MNRAS, 272, 80 (CM95).

Chakrabarti, S. K., \& Titarchuk, L. G., 1995, ApJ, 455, 623.

Davies, M. B., Ruffert, M., Benz, W., \& Müller, E., 1993, A \& A, 272, 430.

Harten, A., 1983, J. Comput. Phys., 49, 357.

Hawley, J. F., Smarr, L. L., \& Wilson, J. R., 1984, ApJ, 277, 296.

Hawley, J. F., Smarr, L. L., \& Wilson, J. R., 1985, ApJ Suppl., 55, 211.

Kang, H., Cen, R., Ostriker, J. P., \& Ryu, D., 1994, ApJ, 428, 1.

Monaghan J. J., Comp. Phys. Repts., 1985, 3, 71.

Monaghan J. J., Ann. Rev. Astron. Astrophys., 1992, 30, 543.

Laguna, P., Miller, W.A., Zurek, W.H., \& Davies, M.B., 1993, ApJ, 410, L83.

Liang, E. P. T., \& Thompson, K. A., 1980, ApJ, 240, 271.

Molteni, D., Chakrabarti, S. K., \& Ryu, D., 1996, in preparation.

Molteni, D., Lanzafame, G., \& Chakrabarti, S. K., 1994, ApJ, 425, 161 (MLC94).

Molteni, D. \& Sponholz, H. 1994, in Journal of Italian Astronomical Society, Vol. 65-N. 4-1994, Ed. G. Bodo \& J.C. Miller

Molteni, D., Sponholz, H., \& Chakrabarti, S. K., 1996, Feb. 1st (MSC96).

Novikov, I., \& Thorne, K. S., 1973, in Black Holes, eds. C. DeWitt and B. DeWitt (Gordon and Breach, New York).

Paczyński, B., \& Bisnovatyi-Kogan, G. , 1981, Acta Astron. 31, 1.

Paczyński, B., \& Wiita, P. J., 1980, A\&Ap, 88, 23.

Ryu, D., Brown, G. L., Ostriker, J. P., and Loeb, A., 1995, ApJ, 452, 364.

Ryu, D., Chakrabarti, S. K., \& Molteni, D. 1996, in preparation. 
Ryu, D., Ostriker, J. P., Kang, H., and Cen, R., 1993, ApJ, 414, 1.

Shakura, N. I., \& Sunyaev, R. A., 1973, A\&A, 24, 337.

Sponholz, H., \& Molteni, D., 1994, MNRAS, 271, 233 (SM94).

Steinmetz, M., \& Müller, E., 1993, A\&Ap, 268, 391.

Thompson, J.M.T. and Stewart, H.B., 1985, Nonlinear Dynamics and Chaos (John Willey \& Sons Ltd.).

Wilson, J. R., 1978, ApJ, 173, 431. 


\section{FIGURE CAPTIONS}

Fig. 1 Comparison of analytical and numerical results in a one-dimensional accretion flow which allows a standing shock. $\mathcal{E}=0.036$ and $\lambda=1.80$ are used. The long and short dashed curves are the results of the TVD and SPH simulations respectively. The solid curve is the analytical result for the same parameters. Upper panel is the mass density in arbitrary units and the lower panel is the Mach number of the flow. Here the flow passes through the outer sonic point (at $x_{o}=27.9$ ), then through a shock (at 7.89), and finally through the inner sonic point (at 2.563).

Fig. 2a-b As in Fig. 1, but for flows without shocks while keeping $\lambda=1.80$. In (a), the flow has $\mathcal{E}=0.07$ and it passes through only the inner sonic point at $x_{i}=2.46$. In (b), the flow has $\mathcal{E}=0.015$ and it passes through only the outer sonic point at $x_{o}=77.615$.

Fig. 3a-b Density contours of (a) the TVD simulation and (b) the SPH simulation in the $x-z$ plane at $T=2 \times 10^{4}$. Same set of contour levels are used. In both the cases the big paraboloidal accretion shock dominates the flow. Also seen are the oblate spheroidal shock near the equatorial plane which forms mainly due to turbulence pressure of the backflow. The parameters used in the calculations are $\mathcal{E}=0.004$ and $\lambda=1.65$ in the outer boundary on the equatorial plane.

Fig. 4a-b As in Fig. 3 but the velocity fields are shown. Super-imposed by solid curves are the locations of the funnel wall (inner curve) and the centrifugal barrier (outer curve) respectively. 

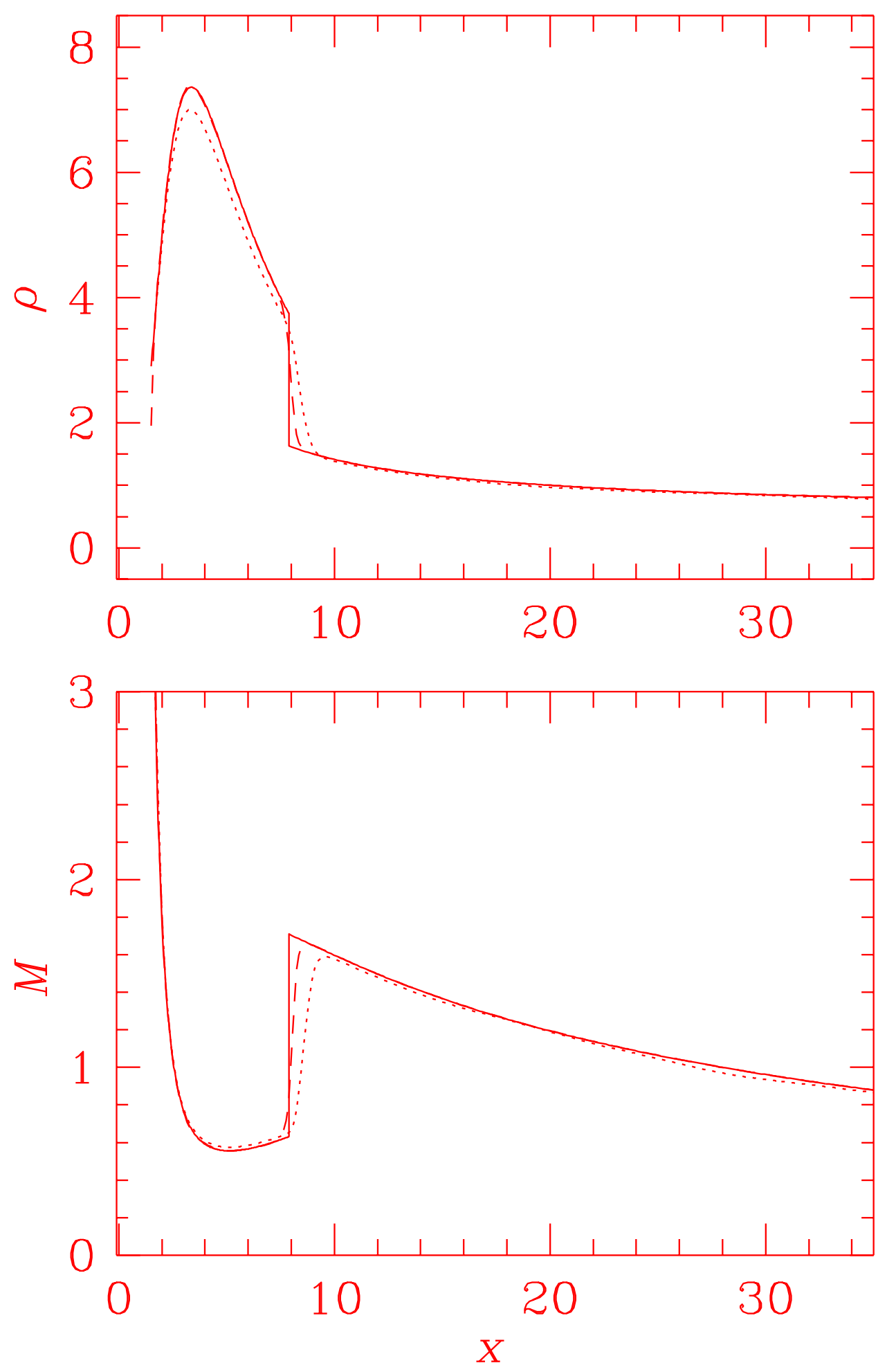

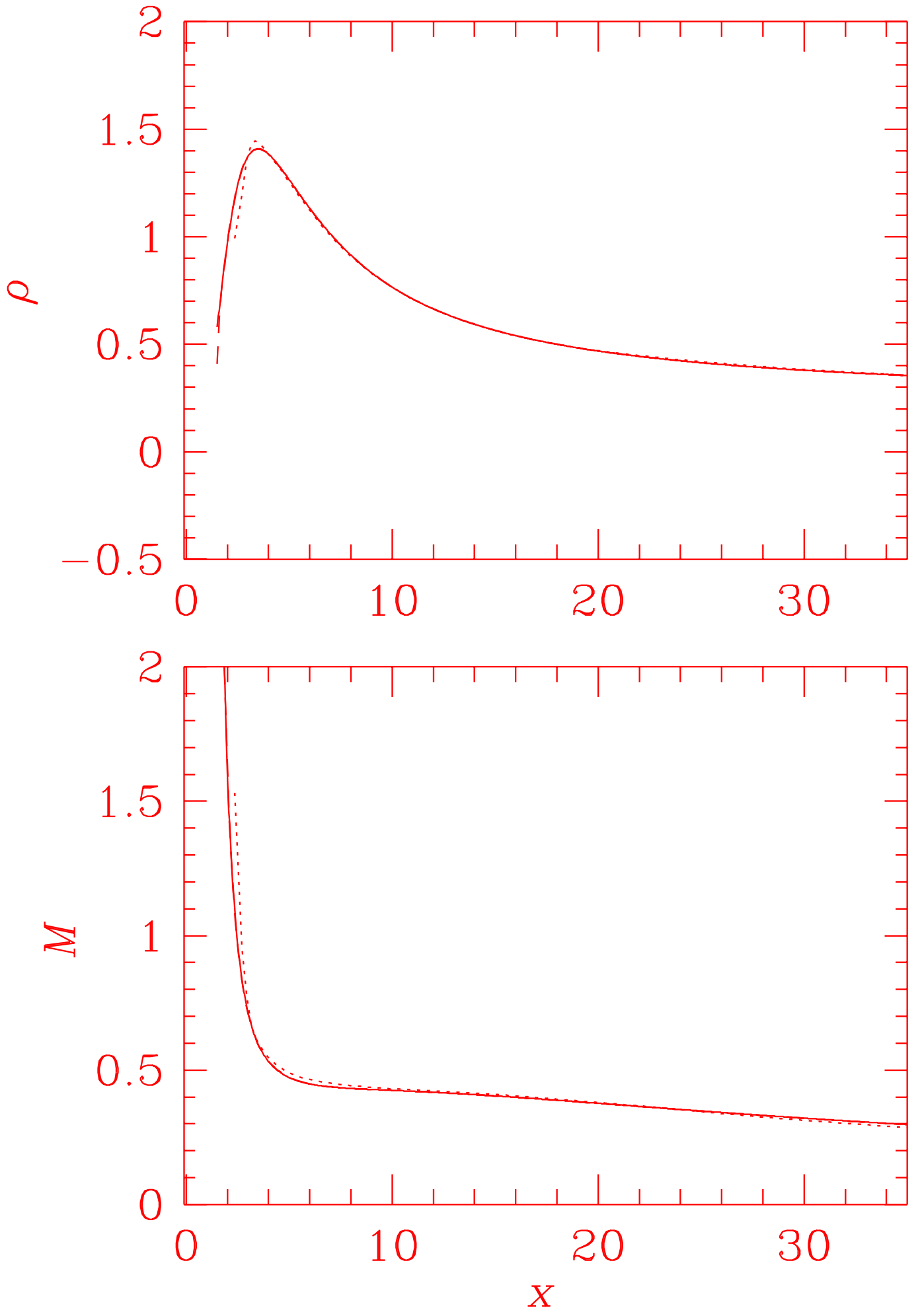

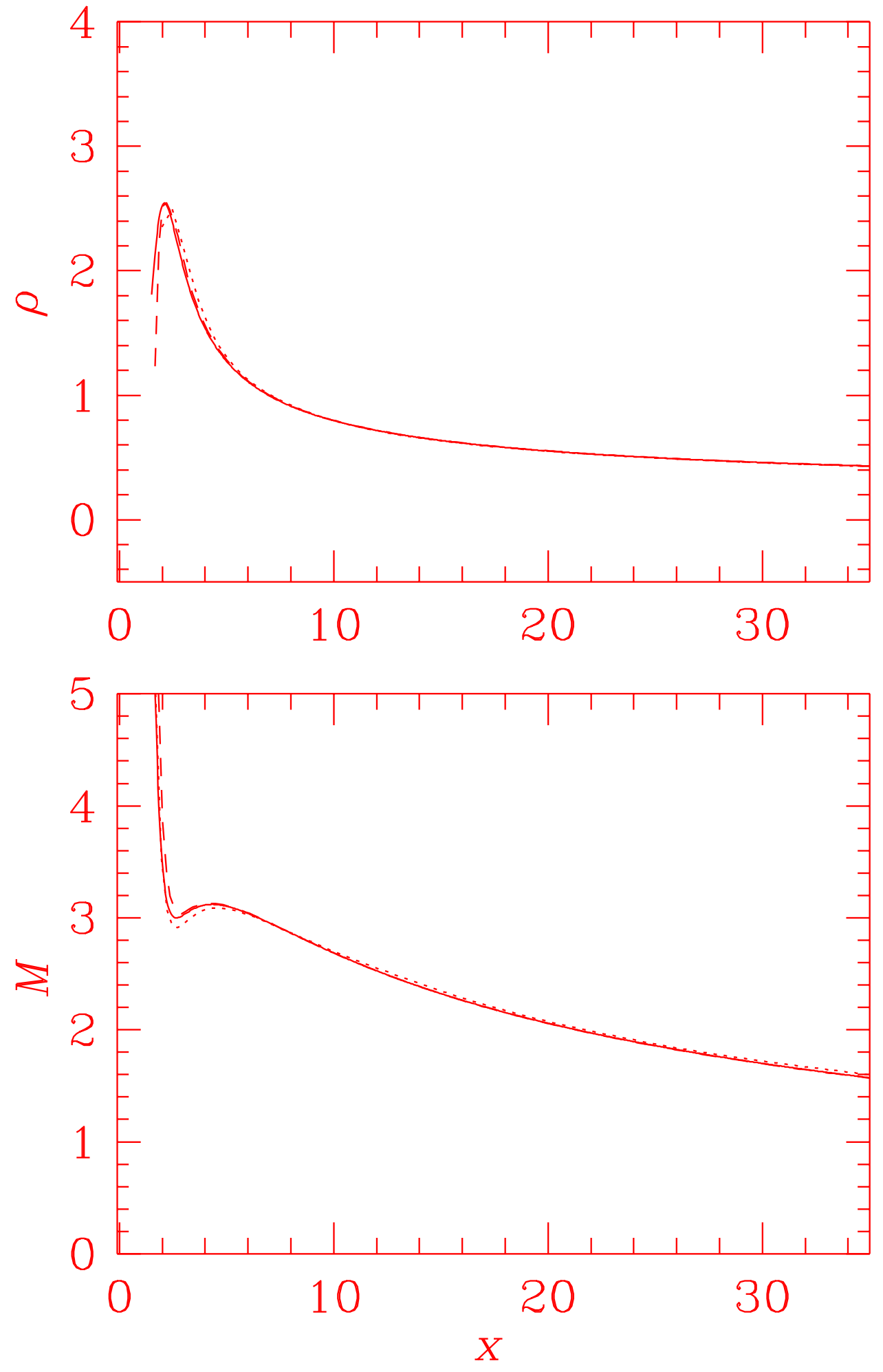


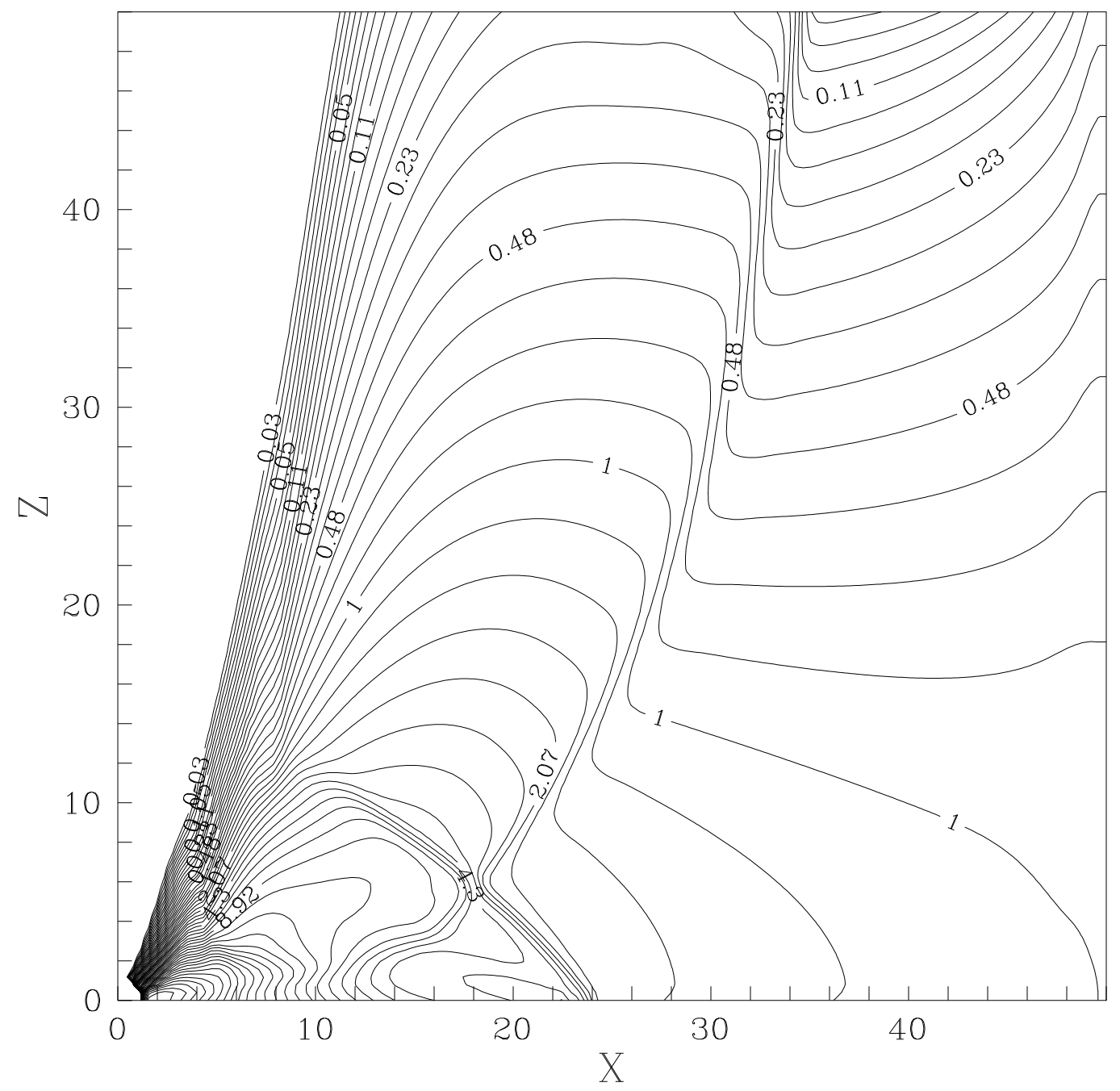




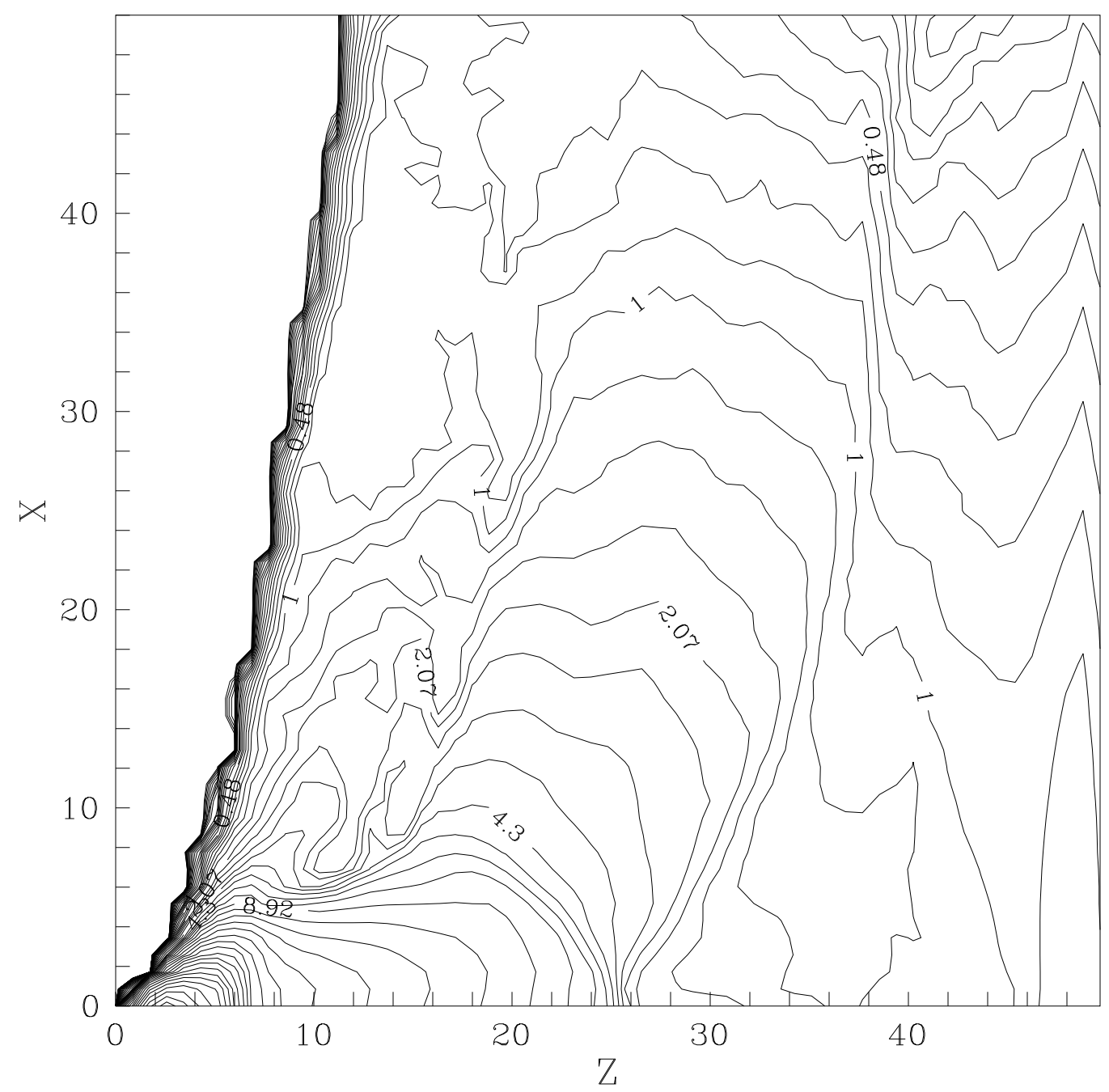




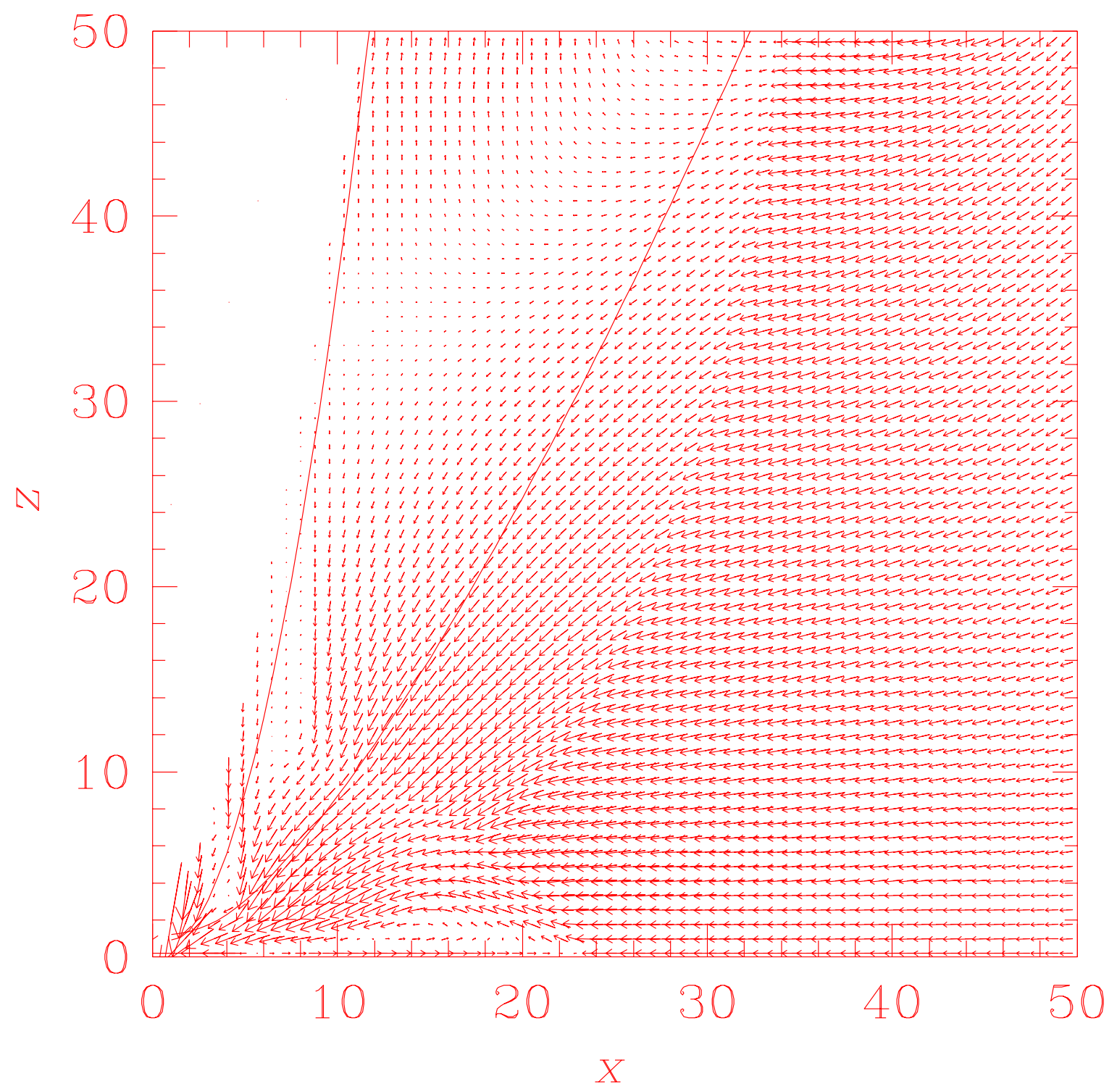




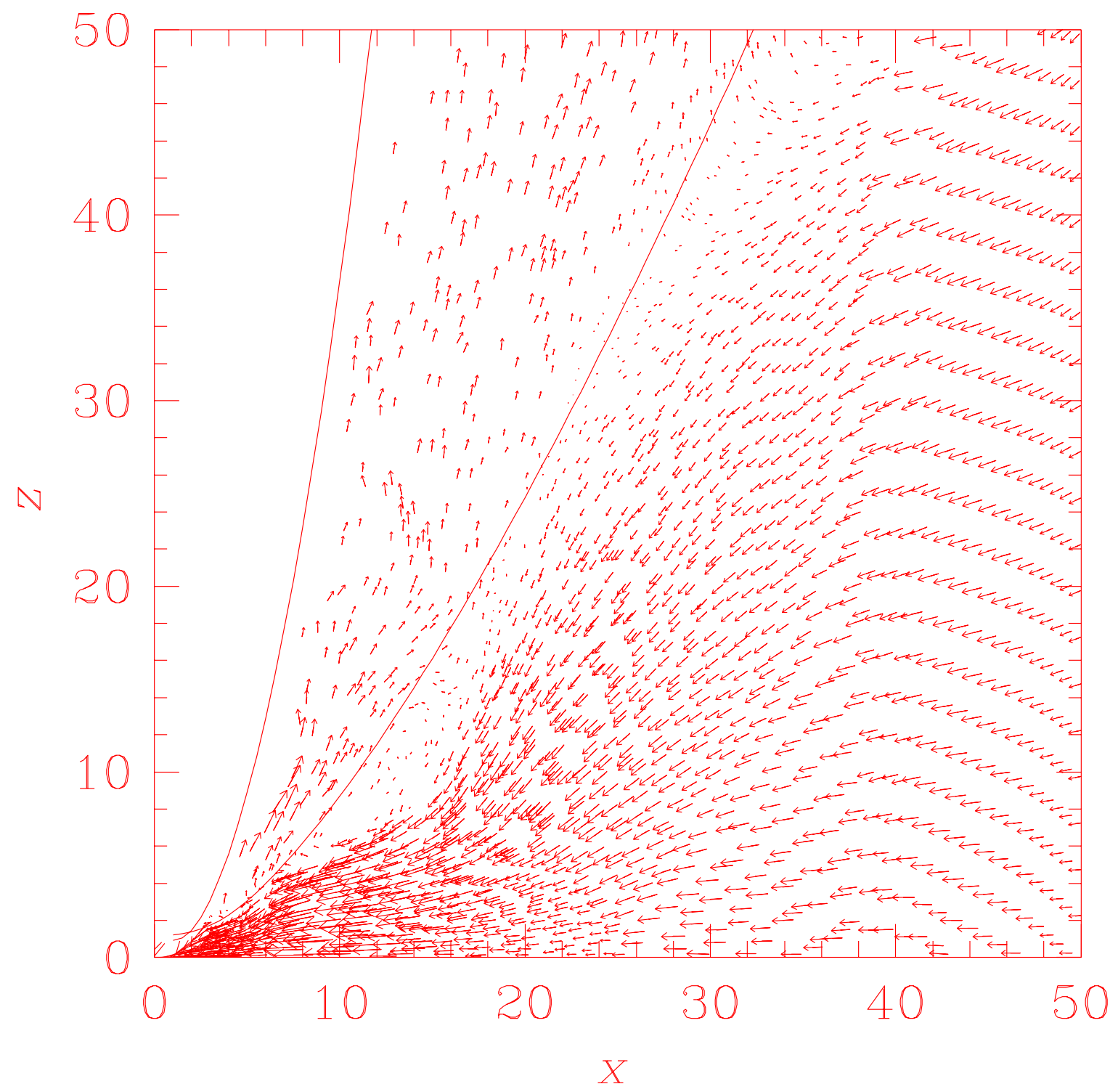

